\title{
The Role of Neutrophil Activation in the Pathogenesis of Kawasaki Disease
}

\author{
Seiichiro Takeshita ${ }^{1 *}$, Yoichi Kawamura², Takashi Kanai ${ }^{2}$, Yusuke Yoshida ${ }^{2}$, Yuki Tsujita ${ }^{2}$ and \\ Shigeaki Nonoyama²
}

${ }^{1}$ Division of Nursing, National Defense Medical College, Tokorozawa, Saitama, Japan

${ }^{2}$ Department of Pediatrics, National Defense Medical College, Tokorozawa, Saitama, Japan

*Corresponding author: Seiichiro Takeshita, Division of Nursing, National Defense Medical College, 3-2 Namiki, Tokorozawa, Saitama 359-8513, Japan, Tel: +81-4-2995-1211; Fax: +81-4-2995-0873; E-mail: takeshit@ndmc.ac.jp

Received date: December 14, 2017; Accepted date: January 1, 2018; Published date: January 4, 2018

Citation: Takeshita S, Kawamura Y, Kanai T, Yoshida Y, Tsujita Y, et al. (2018) The Role of Neutrophil Activation in the Athogenesis of Kawasaki Disease. Pediatric Infect Dis Vol 3, No 1: 1.

Copyright: @ 2018 Takeshita S, et al. This is an open-access article distributed under the terms of the Creative Commons Attribution License, which permits unrestricted use, distribution, and reproduction in any medium, provided the original author and source are credited.

\begin{abstract}
In the acute phase of Kawasaki disease (KD), there is an increase in the number of circulating neutrophils, including toxic neutrophils, which are caused by a morphological change. The functions of the neutrophils are also activated, leading to the excessive production of reactive oxygen species and elastase, which may induce endothelial cell (EC) injury. Histological findings demonstrate that numerous neutrophils infiltrate into coronary artery lesions (CALS) during the early phase of KD. Thus, it is suggested that neutrophil-mediated EC injury may be involved in the formation of CALs in KD patients. In this review, we focus on the role of neutrophil activation in the pathogenesis of KD vasculitis and discuss the methods of treatment for neutrophil-mediated EC injury.
\end{abstract}

Keywords: Kawasaki disease; Neutrophil; Coronary artery lesion; Endothelial cell

\section{Introduction}

Human polymorphonuclear neutrophils play a key role in the host defense by ingesting and destroying infectious agents. Neutrophils contain a number of proteases in their own granules; the proteases are also involved in the physiological processes of matrix proteolysis, which include intercellular migration, tissue remodeling, and repair $[1,2]$. However, if the activity of the proteases secreted from neutrophils is uncontrolled and excessive in conditions such as severe inflammatory disease, they can degrade the extracellular matrix, thus leading to the destruction of tissue. Neutrophil elastase, which is stored in an active form within the azurophilic granules, is an enzyme from the class of proteases that break down proteins. Since elastase is the strongest serine protease, this enzyme is reported to play the main role in the neutrophilmediated injury of endothelial cells (ECs) [1]. EC injury mediated by activated neutrophils has been reported to occur in systemic inflammatory response syndrome (SIRS), adult respiratory distress syndrome (ARDS) and multiple organ failure (MOF) [3].

Kawasaki disease (KD) is an acute febrile illness that predominantly affects infants and children [4]. This disease is well known to be a type of systemic vasculitis, and the most critical complication is the occurrence of a coronary artery lesion (CAL), which may lead to myocardial infarction [5]. Although high-dose intravenous immunoglobulin (IVIG) is the standard initial therapy for acute KD $[6,7]$, approximately $10-20 \%$ of KD patients are resistant to IVIG therapy. IVIG-resistant patients are at higher risk of developing CAL than IVIG-responsive patients $[6,7]$. Approximately $3 \%$ of KD patients are reported to have cardiac sequelae, such as coronary dilatation and aneurysm [8]. Although KD is the primary cause of acquired heart disease in children in developed countries, the etiology remains unclear. KD patients have high levels of circulating proinflammatory cytokines and soluble adhesion molecules, indicating that the immune system is markedly activated in this disease [9]. In the acute phase of KD, circulating neutrophils are known to increase in number and are functionally activated, which causes the excessive production of reactive oxygen species (ROS) [10] and elastase [11]. Furthermore, the elastase levels in the acute phase of KD are reported to be higher than in the acute phase of sepsis [11]. Thus, it is suggested that neutrophil-mediated EC injury may contribute to the formation of CALs in patients with $K D$. In the present review, we focus on the role of neutrophil activation in the pathogenesis of KD vasculitis and discuss the therapeutic methods for treating neutrophil-mediated EC injury.

\section{The increased number of neutrophils and the} morphological changes of neutrophils in acute KD

In the acute phase of KD, the white blood cell count is elevated and consists mainly of polymorphonuclear neutrophils with a shift to the left. Morphologically, many toxic neutrophils, which are characterized by cytotoxic vacuolization, swelling and toxic granulation with or without Döhle bodies, are seen in the circulation $[12,13]$. Furthermore, the percentage of toxic neutrophils in the acute phase of KD tends to be higher in patients with CAL than in those without CAL [12]. Kobayashi et 
al. reported a risk-scoring system for predicting IVIG resistance, in which the percentage of neutrophils ( $\geq 80 \%)$ is included as a factor [14]. Recently, we reported that an increased neutrophilto-lymphocyte ratio can independently predict IVIG resistance in KD patients $[15,16]$. Thus, it is suggested that neutrophilia with toxic neutrophils may be a marker that reflects the severity of KD.

\section{The role of neutrophil activation in the pathogenesis of $K D$ vasculitis}

Activated neutrophil-mediated EC injury in KD vasculitis: Histologically, the edematous changes of KD start in the media on the sixth to eighth day of illness, and inflammatory cells infiltrate into the intima and adventitia, forming panvasculitis across all layers of the vessel wall, by the tenth day of illness [17]. Takahashi et al. demonstrated the infiltration of numerous neutrophils (anti-elastase-positive neutrophils) in CALs during the early phase of KD (seventh to ninth day of illness), before the infiltration of macrophages and lymphocytes, suggesting that neutrophils are involved in the initial damage that occurs to the coronary arteries [18].

The function of neutrophils in the early stage of KD is reported to be enhanced with a marked increase in ROS [10]. The plasma levels of neutrophil elastase and myeloperoxidase (MPO) were reported to be increased in the acute phase of KD [11]. Furthermore, the plasma levels of neutrophil elastase were reported to be higher in patients with CAL than in those without CAL [19]. Biezeveld et al. reported that the plasma levels of neutrophil elastase remained high from the acute phase of KD throughout the convalescent phase (three months after the onset), suggesting that neutrophil activation may be sustained for longer than was previously assumed [20]. Thus, activated neutrophil-mediated EC injury is thought to be involved in the pathogenesis of $K D$ vasculitis.

The up-regulation of the adhesion molecule expression of neutrophils in acute KD: Hamamichi et al. reported that circulating neutrophils abundantly express vascular endothelial growth factor (VEGF) in the acute phase of KD, suggesting that neutrophil-derived VEGF may contribute to the vascular injury and remodeling observed in $K D$ vasculitis [21]. The $C D 11 b$ expression of circulating neutrophils was increased in the acute phase of KD and was positively correlated with the serum levels of IL-6, IL-10, and granulocyte colony-stimulating factor (G-CSF) [22]. The expression of $C D 64(F c \gamma R)$ of neutrophils is reported to dramatically increase in the acute phase of KD and to decrease after IVIG infusion $[23,24]$. Since neutrophils primed in vitro with IFN- $\gamma$ and G-CSF can induce the expression of CD64 $[25,26]$, the up-regulation of CD64 may reflect the activation of neutrophils by inflammatory cytokines. The serum levels of G-CSF in IVIGresistant patients were found to be significantly higher than those in IVIG-responsive patients, suggesting that the G-CSF stimulation of neutrophils may be an important risk factor for KD [27]. Circulating platelet-neutrophil aggregates are reported to be present at higher rates in patients with CAL than in those without CAL, suggesting that the platelet-neutrophil interaction may play a significant role in $\mathrm{KD}$ vasculitis by promoting the migration and accumulation of neutrophils into vascular tissues [28].

The increased production of nitric oxide and nitric oxide synthase in acute KD: Nitric oxide (NO) is synthesized by nitric oxide synthase (NOS) and plays diverse roles in the physiology and pathophysiology of the cardiovascular system $[29,30]$. Although NO plays a vasoprotective role by inhibiting platelet aggregation, leukocyte adherence and vascular smooth muscle cell proliferation, the release of a large amount of NO can cause tissue damage and arterial wall degeneration. During the early stage of acute $K D$, it is reported that circulating neutrophils expressed both NO and NOS, and that the production level of inducible NOS (iNOS) in neutrophils was higher in patients with CAL than in those without CAL [31,32]. Thus, it is suggested that NO, which is synthesized by iNOS in neutrophils, may play an important role in the formation of CALs in KD vasculitis.

Delayed apoptosis of circulating neutrophils in acute KD: Human neutrophils are constitutively programmed to undergo apoptosis, and their life span is short. Since the prolonged survival of activated neutrophils has autotoxic potential due to the release of proteases and ROS, neutrophil apoptosis may represent a suitable mechanism for resolving inflammation. We previously reported that the apoptosis of neutrophils is inhibited during the acute phase of $K D$, and that an increase in the number of peripheral neutrophils may be associated with the delayed apoptosis of neutrophils [33]. Furthermore, IVIG can promote apoptosis in activated neutrophils in vitro by an oxygen-dependent pathway via FcpRII and III [34]. Actually, in KD patients, IVIG therapy is reported to decrease the number of circulating neutrophils by accelerating their apoptosis [35]. Thus, it is suggested that the prolongation of the life span of activated neutrophils in circulation may be involved in the pathogenesis of KD vasculitis, and that IVIG therapy may have a protective effect on activated neutrophil-mediated EC injury by inducing apoptosis in neutrophils.

The inhibitory effect of protease inhibitor on neutrophilmediated EC injury in KD vasculitis: Although IVIG is the goldstandard therapy for acute KD, CAL formation cannot be completely prevented by IVIG alone [6,7]. Thus, it is believed that intensive initial therapy combined with primary IVIG and other anti-inflammatory drugs may reduce IVIG resistance and CAL formation in high-risk KD patients. Since the activated neutrophil-mediated EC injury is thought to be involved in the pathogenesis of KD vasculitis, the neutrophils may be a potential target of therapy in KD. Elastase is thought to be the primary agent of neutrophil-mediated EC injury [1]. Ulinastatin (urinary trypsin inhibitor, UTI), a serine protease inhibitor, inhibits neutrophil-mediated EC injury in vitro, by inactivating the extracellular elastase secreted from the neutrophils and suppressing the production of intracellular elastase in them [36]. UTI has been shown to have clinical applications in the treatment of inflammatory disorders such as pancreatitis, circulatory shock, septic shock, ARDS and disseminated intravascular coagulation (DIC) [37]. Recently, we reported that initial UTI therapy combined with IVIG reduced the proportion of $K D$ patients requiring additional rescue treatment and the occurrence of CAL in comparison to IVIG alone [38]. Thus, UTI in 
combination with IVIG may be a candidate for the initial treatment of KD.

\section{Conclusion}

A schematic illustration of neutrophil-mediated EC injury in KD vasculitis and the mechanisms of action of IVIG and UTI are shown in the Figure 1 . In the acute phase of KD, circulating neutrophils are functionally activated by inflammatory mediators such as cytokines. The survival of the activated neutrophils, which produce excess amounts of proteases (elastase and MPO), ROD and NO, is prolonged because their apoptosis is delayed which may induce EC injury and neutrophil transmigration and contribute to the pathogenesis of KD vasculitis. Thus, the activated neutrophils may be a therapeutic target in KD. IVIG may not only inhibit the production of cytokines by monocytes and macrophages, but also promote the apoptosis of activated neutrophils. On the other hand, UTI may inactivate the elastase secreted from neutrophils and suppress the elastase production in them. Thus, it is suggested that both drugs protect ECs against neutrophil-mediated injury in KD vasculitis via different mechanisms. Since activated neutrophilmediated EC injury is thought to be involved in the pathogenesis of KD, the clarification of its mechanism may lead to the improved treatment of this disease.

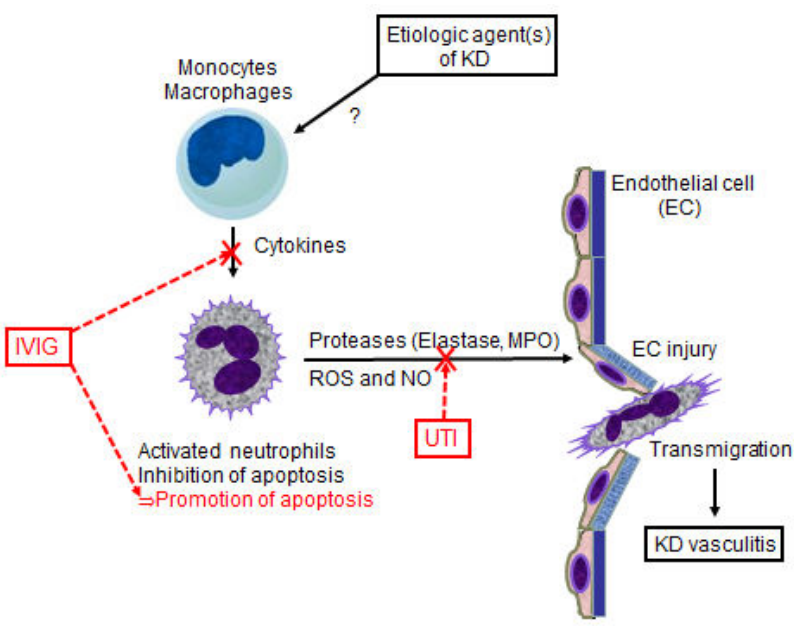

Figure 1: Neutrophil-medicated EC injury in KD vasculitis and mechanisms of action of IVIG and UTI. (EC: Endothelial Cell; IVIG: Intravenous Immunoglobulin; KD: Kawasaki Disease; MPO: Myeloperoxidase; NO: Nitric Oxide; ROS: Reactive Oxygen Species; UTI: Ulinastatin).

\section{Funding}

This study was supported by grants from the Grant-in-Aid for Scientific Research (KAKENHI, 16K10082). The funders had no role in study design, data collection and analysis, decision to publish, or preparation of the manuscript.

\section{Competing Interests}

The authors have declared that no competing interests exist.

\section{References}

1. Smedly LA, Tonnesen MG, Sandhaus RA, Haslett C, Guthrie LA, et al. (1986) Neutrophil-mediated injury to endothelial cells. Enhancement by endotoxin and essential role of neutrophil elastase. J Clin Invest 77: 1233-1243.

2. Owen CA, Campbell EJ (1999) The cell biology of leukocytemediated proteolysis. J Leukoc Biol 65: 137-150.

3. Chen X, Christou NV (1996) Relative contribution of endothelial cell and polymorphonuclear neutrophil activation in their interactions in systemic inflammatory response syndrome. Arch Surg 131: 1148-1154.

4. Kawasaki T, Kosaki F, Okawa S, Shigematsu I, Yanagawa H (1974) A new infantile acute febrile mucocutaneous lymph node syndrome (MLNS) prevailing in Japan. Pediatrics 54: 271-276.

5. Kato H, Koike S, Yamamoto M, Ito Y, Yano E (1975) Coronary aneurysms in infants and young children with acute febrile mucocutaneous lymph node syndrome. J Pediatr 86: 892-898.

6. Newburger JW, Takahashi M, Beiser AS, Burns JC, Bastian J, et al. (1991) A single intravenous infusion of gamma globulin as compared with four infusions in the treatment of acute Kawasaki syndrome. New Engl J Med 324: 1633-1639.

7. Nakamura $Y$, Yashiro $M$, Uehara R, Sadakane A, Tsuboi $S$, et al. (2012) Epidemiologic features of Kawasaki disease in Japan: results of the 2009-2010 nationwide survey. J Epidemiol 22: 216221.

8. Nakamura Y (2016) Update on Nationwide surveys and epidemiologic characteristics of Kawasaki disease in Japan. KD 79-84.

9. Burns JC, Glode MP (2004) Kawasaki syndrome. Lancet 364: 533-544.

10. Niwa Y, Sohmiya K (1984) Enhanced neutrophilic functions in mucocutaneous lymph node syndrome, with special reference to the possible role of increased oxygen intermediate generation in the pathogenesis of coronary thromboarteritis. J Pediatr 104: 56-60.

11. Takeshita S, Nakatani K, Kawase H, Seki S, Yamamoto M, et al. (1999) The role of bacterial lipopolysaccharide-bound neutrophils in the pathogenesis of Kawasaki disease. J Infect Dis 179: 508-512.

12. Takeshita S, Sekine I, Fujisawa T, Yoshioka S (1990) Studies of peripheral blood toxic neutrophils as a predictor of coronary risk in Kawasaki disease-the pathogenetic role of hematopoietic colony-stimulating factors (GM-CSF, G-CSF). Acta Paediatr Jpn 32: 508-514.

13. Takeshita S, Kanai T, Kawamura Y (2016) Kawasaki Disease: Current understanding of the mechanism and evidence-based Treatment. Springer.

14. Kobayashi T, Inoue Y, Takeuchi K, Okada Y, Tamura K, et al. (2006) Prediction of intravenous immunoglobulin unresponsiveness in patients with Kawasaki disease. Circulation 113: 2606-2612.

15. Kawamura Y, Takeshita S, Kanai T, Yoshida Y, Nonoyama S (2016) The Combined Usefulness of the Neutrophil-to-Lymphocyte and Platelet-to-Lymphocyte Ratios in Predicting Intravenous Immunoglobulin Resistance with Kawasaki Disease. J Pediatr 178: 281-284.

16. Takeshita S, Kanai T, Kawamura Y, Yoshida Y, Nonoyama S (2017) A comparison of the predictive validity of the combination of the neutrophil-to-lymphocyte ratio and platelet-to-lymphocyte ratio 
and other risk scoring systems for intravenous immunoglobulin (ivig)-resistance in Kawasaki disease. PLoS One 12: e0176957.

17. Oharaseki T, Yokouchi Y, Enomoto Y, Takahashi K (2016) Histopathology of coronary arthritis in acute Kawasaki disease and murine systemic vasculitis induced by Candida albicans cell wall polysaccharide. KD 11-16.

18. Takahashi K, Oharaseki T, Naoe S, Wakayama M, Yokouchi Y (2005) Neutrophilic involvement in the damage to coronary arteries in acute stage of Kawasaki disease. Pediatr Int 47: 305-310.

19. Senzaki H, Masutani $S$, Kobayashi J, Kobayashi T, Nakano H, et al. (2001) Circulating matrix metalloproteinases and their inhibitors in patients with Kawasaki disease. Circulation 104: 860-863.

20. Biezeveld MH, van Mierlo G, Lutter R, Kuipers IM, Dekker T, et al. (2005) Sustained activation of neutrophils in the course of Kawasaki disease: an association with matrix metalloproteinases. Clin Exp Immunol 141: 183-188.

21. Hamamichi $\mathrm{Y}$, Ichida $\mathrm{F}, \mathrm{Yu} \mathrm{X}$, Hirono $\mathrm{KI}$, Uese $\mathrm{KI}$, et al. (2001) Neutrophils and mononuclear cells express vascular endothelial growth factor in acute Kawasaki disease: its possible role in progression of coronary artery lesions. Pediatr Res 49: 74-80.

22. Kobayashi T, Kimura H, Okada Y, Inoue Y, Kobayashi T, et al. (2007) Increased CD11b expression on polymorphonuclear leucocytes and cytokine profiles in patients with Kawasaki disease. Clin Exp Immunol 148: 112-118.

23. Nakatani K, Takeshita S, Tsujimoto H, Kawamura Y, Kawase H, et al. (1999) Regulation of the expression of Fc gamma receptor on circulating neutrophils and monocytes in Kawasaki disease. Clin Exp Immunol 117: 418-422.

24. Hokibara S, Kobayashi N, Kobayashi K, Shigemura T, Nagumo H, et al. (2016) Markedly elevated CD64 expression on neutrophils and monocytes as a biomarker for diagnosis and therapy assessment in Kawasaki disease. Inflamm Res 65: 579-585.

25. Cassatella MA, Flynn RM, Amezaga MA, Bazzoni F, Vicentini F, et al. (1990) Interferon gamma induces in human neutrophils and macrophages expression of the mRNA for the high affinity receptor for monomeric IgG (Fc gamma R-I or CD64). Biochem Biophys Res Commun 170: 582-588.

26. Repp R, Valerius T, Sendler A, Gramatzki M, Iro H, et al. (1991) Neutrophils express the high affinity receptor for IgG (Fc gamma RI, CD64) after in vivo application of recombinant human granulocyte colony-stimulating factor. Blood 78: 885-889.
27. Abe J, Ebata R, Jibiki T, Yasukawa K, Saito H, et al. (2008) Elevated granulocyte colony-stimulating factor levels predict treatment failure in patients with Kawasaki disease. J Allergy Clin Immunol 122: $1008-1013$

28. Ueno K, Nomura Y, Morita Y, Eguchi T, Masuda K, et al. (2015) Circulating platelet-neutrophil aggregates play a significant role in Kawasaki disease. Circ J 79: 1349-1356.

29. Beckman JS, Beckman TW, Chen J, Marshall PA, Freeman BA (1990) Apparent hydroxyl radical production by peroxynitrite: implications for endothelial injury from nitric oxide and superoxide. Proc Natl Acad Sci USA 87: 1620-1624.

30. Kibbe M, Billiar T, Tzeng E (1999) Inducible nitric oxide synthase and vascular injury. Cardiovasc Res 43: 650-657.

31. Yu X, Hirono KI, Ichida F, Uese K, Rui C, et al. (2004) Enhanced iNOS expression in leukocytes and circulating endothelial cells is associated with the progression of coronary artery lesions in acute Kawasaki disease. Pediatr Res 55: 688-694.

32. Yoshimura K, Tatsumi K, Iharada A, Tsuji S, Tateiwa A, et al. (2009) Increased nitric oxide production by neutrophils in early stage of Kawasaki disease. Eur J Pediatr 168: 1037-1041.

33. Tsujimoto H, Takeshita S, Nakatani K, Kawamura Y, Tokutomi T, et al. (2001) Delayed apoptosis of circulating neutrophils in Kawasaki disease. Clin Exp Immunol 126: 355-364.

34. Takeshita S, Tsujimoto H, Nakatani K (2005) Intravenous immunoglobulin preparations promote apoptosis in lipopolysaccharide-stimulated neutrophils via an oxygendependent pathway in vitro. APMIS 113: 269-277.

35. Tsujimoto H, Takeshita S, Nakatani K, Kawamura Y, Tokutomi T, et al. (2002) Intravenous immunoglobulin therapy induces neutrophil apoptosis in Kawasaki disease. Clin Immunol 103: 161-168.

36. Nakatani K, Takeshita S, Tsujimoto H, Kawamura Y, Sekine I (2001) Inhibitory effect of serine protease inhibitors on neutrophilmediated endothelial cell injury. J Leukocyte Biol 69: 241-247.

37. Inoue K, Takano H (2010) Urinary trypsin inhibitor as a therapeutic option for endotoxin-related inflammatory disorders. Expert Opin Investig Drugs 19: 513-520.

38. Kanai T, Ishiwata T, Kobayashi T, Sato H, Takizawa M, et al. (2011) Ulinastatin, a urinary trypsin inhibitor, for the initial treatment of patients with Kawasaki disease: a retrospective study. Circulation 124: $2822-2828$ 